\title{
Risk factors for retear of large/massive rotator cuff tears after arthroscopic surgery: an analysis of tearing patterns
}

Hisao Shimokobe ${ }^{1}$, Masafumi Gotoh ${ }^{3 *}$, Hirokazu Honda ${ }^{3}$, Hidehiro Nakamura', Yasuhiro Mitsui ${ }^{3}$, Tatsuyuki Kakuma ${ }^{2}$, Takahiro Okawa ${ }^{3}$ and Naoto Shiba ${ }^{1}$

\begin{abstract}
Background: Previous studies have evaluated the risk factors for retear of large/massive rotator cuff tears (RCTs) that were treated arthroscopically; however, most studies did not evaluate tear patterns. The present study hypothesized that postoperative risk factors are affected by the tearing patterns in large/massive cuff tears in patients undergoing arthroscopic rotator cuff repair (ARCR).

Methods: One hundred fifty patients with large/massive cuff tears underwent ARCR at our institution. Of these, 102 patients were enrolled in this study, with an average symptom duration of $36.3 \pm 43.9$ months and average age of 63 . $9 \pm 9.4$ years. According to the arthroscopic findings and magnetic resonance imaging (MRI), the 102 patients were divided into three groups based on the tendon location: anterosuperior tears ( $N=59$, group $A S$ ), posteosuperior tears ( $N=21$, group PS), and anteroposterior-extending tears ( $N=22$, group APE). Functional outcome was evaluated preoperatively and postoperatively using the Japanese Orthopedic Association (JOA) score and the University of California, Los Angeles (UCLA) score. Retear was evaluated with MRI at a minimum of 1 year after surgery, using Sugaya's classification; Types IV and $\vee$ were considered postoperative retears. Factors affecting postoperative retear were examined with univariate and multivariate analyses.
\end{abstract}

Results: JOA/UCLA scores significantly improved postoperatively in the three groups ( $P<0.01 \mathrm{for}$ all). Postoperative retear was noted in 26 of 102 patients (25.5\%) in this series: 10 patients in group AS (16.9\%), 9 in group PS (42.9\%), and 7 in group APE (31.8\%). The retear rate was significantly higher in group PS than in the other two groups $(P=0.02)$. Multivariate analysis showed that decreased preoperative active external rotation range was a unique risk factor for postoperative retear in the PS and APE groups (95\% confidence interval: $0.02-0.18$, cut-off value: $25^{\circ}$, with an area under the curve of $0.90, P=0.0025)$.

Conclusions: Although multivariate analysis failed to detect significant risk factor for retear in patients with anterosuperior large/massive cuff tears who undergo ARCR, it demonstrated that active external rotation less than $25^{\circ}$ before surgery is a significant risk factor in those with posterosuperior large/massive tears. This study may help surgeons understand the results of arthroscopic surgery in patients with large/massive tears.

Keywords: Tearing pattern, Arthroscopic rotator cuff repair, Postoperative retear

\footnotetext{
* Correspondence: gomasa@med.kurume-u.ac.jp

${ }^{3}$ Department of Orthopaedic Surgery, Kurume University Medical Center,

155-1 Kokubu-machi Kurume, Fukuoka 839-0863, Japan

Full list of author information is available at the end of the article
}

(c) The Author(s). 2017 Open Access This article is distributed under the terms of the Creative Commons Attribution 4.0 International License (http://creativecommons.org/licenses/by/4.0/), which permits unrestricted use, distribution, and reproduction in any medium, provided you give appropriate credit to the original author(s) and the source, provide a link to the Creative Commons license, and indicate if changes were made. The Creative Commons Public Domain Dedication waiver (http://creativecommons.org/publicdomain/zero/1.0/) applies to the data made available in this article, unless otherwise stated. 


\section{Background}

Arthroscopic rotator cuff repair (ARCR) produces good clinical results, although retear is a significant concern after surgery. Compared with small- and middle-sized rotator cuff tears (RCTs), the retear rate is relatively high in large and massive tears, even if the tear is completely covered during surgery [1-4]; some authors reported that the retear rate was $40-94 \%$ in these tears [3-6].

A number of studies have consistently sought to determine the risk factors for postoperative retear in large and massive cuff tears. For example, a recent systematic review reported that the risk factors for retear after ARCR in RCTs included age, tear size, fatty degeneration (FD), the number of tendons involved, acromiohumeral interval, surgical technique, and bone mineral density [7]. One study used a multivariate regression analysis to demonstrate that preoperative FD of the infraspinatus was the most independent predictor of retear in large and massive RCTs in patients who underwent ARCR [8]. Kim et al. [9] reported that the extent of retraction was importantly associated with retear after surgery.

Based on the tendon location involved, large and massive RCTs are classified into three types: anterosuperior tears, posteosuperior tears, and anteroposterior-extending tears. However, previous studies collectively examined large and massive RCTs without sub-dividing the tear pattern as described above. Therefore, the purposes of the present study were to evaluate risk factors affecting postoperative retear in each group. We hypothesized that in large and massive tears, the risk factors for postoperative retear differ among the groups, when sub-divided by the tear pattern.

\section{Methods}

The patients provided informed consent, and this retrospective study was approved by the authorized institutional review board at the Ethical Committee of Kurume University (\#12333).

\section{Patients}

Between April 2005 and August 2013, 150 patients with cuff tears defined as large or massive [6] underwent ARCR in our institution. The inclusion criteria were (1) individuals who had large or massive rotator cuff tears that repaired completely during surgery, (2) those who were available for evaluation of function and magnetic resonance imaging (MRI) preoperatively and at a minimum of 1 year after surgery, and (3) those who underwent an appointed postoperative rehabilitation program. The exclusion criteria were (1) individuals with advanced glenohumeral arthritis or fractures around the shoulder, (2) those who underwent open repair, partial repair, revision surgeries, or any previous shoulder surgery, (3) those who had MRI film without the "Scapula - Y" view on the sagittal-oblique plane, (4) those who refused to undergo postoperative clinical assessment and MRI, and (5) those who had preoperative stiffness that showed less than $100^{\circ}$ in passive elevation or $10^{\circ}$ in external rotation [10]. Consequently, 102 patients were enrolled in this study.

According to the arthroscopic and MRI findings, the 102 patients were divided into three groups based on the tendon location: anterosuperior tears [11] in the subscapularis and the supraspinatus, in which the tear extended from the lesser tuberosity to the superior facet ( $N=59$, group AS); posteosuperior tears in the supraspinatus and the infraspinatus/teres minor, in which the tear extended from the superior facet to the middle or inferior facet $(N=21$, group PS); and anteroposteriorextending tears, in which the tear extended from the lesser tuberosity to the middle or inferior facet $(N=22$, group APE). When large or massive tears were evaluated according to the classification of DeOrio and Cofield [12], there were 56 patients with a large tear (94.9\%) and 3 patients with a massive tear (5.1\%) in Group AS, 19 patients with a large tear (90.5\%) and 3 patients with a massive tear $(9.5 \%)$ in group PS, and 15 patients with a large tear (58.2\%) and 7 patients with massive tears (31.8\%) in group APE. There was no statistical difference in demographic data among the three groups, except for the incidence of hypertension and distribution of massive tears. Details of the patients' characteristics are shown in Table 1.

\section{Surgical procedure}

Arthroscopic surgery was indicated when successful non-operative treatment, such as anti-inflammatory medications, physical therapy, subacromial or glenohumeral injections of corticosteroids or hyaluronic acid, or activity modification, was not achieved within 3 months of the first visit.

ARCR was conducted with the patient in the beach chair position under general anesthesia. At first, a glenohumeral examination was performed through a posterior portal and then transferred to the subacromial bursa. After making a lateral portal, we identified the ruptured tendon edge and evaluated its flexibility by grasping the tendon and reducing the edge to the original footprint. Capsular release was conducted from the anterior, anterolateral, or posterolateral portal; if needed, tenotomy of the long head biceps was performed. The method of cuff repair was selected based on the operative findings, tendon mobility, and tear condition with a single-row, double-row, or suture bridge technique (Table 1).

\section{Rehabilitation protocol}

Postoperatively, the patient's arm was fixed into a sling with an abduction pillow. Passive range of motion (ROM) exercises of the shoulder were conducted 4 days after surgery. Active ROM exercises and isometric exercise were 
Table 1 Patient demographic data

\begin{tabular}{|c|c|c|c|}
\hline & Group AS $(N=59)$ & Group PS $(N=21)$ & Group APE $(N=22)$ \\
\hline Age (years) & $62.8 \pm 10.6(39-82)$ & $64.9 \pm 8.8(43-78)$ & $66.4 \pm 5.8(54-76)$ \\
\hline Sex: male (\%)/female (\%) & $30(50.1 \%) / 29(49.1 \%)$ & $13(62 \%) / 8(38 \%)$ & $13(59 \%) / 9(41 \%)$ \\
\hline Side: right (\%)/left (\%) & $43(72.9 \%) / 16(27.1 \%)$ & $11(52.4 \%) / 10(47.6 \%)$ & $19(86.4 \%) / 3(13.6 \%)$ \\
\hline Symptom duration (week) & $30.8 \pm 30.3(4-156)$ & $53.9 \pm 69.9(4-275)$ & $34.1 \pm 40.9(2-150)$ \\
\hline Trauma (\%) & $34(57.6 \%)$ & $16(76.2 \%)$ & $12(54.5 \%)$ \\
\hline \multicolumn{4}{|l|}{ Complication } \\
\hline Diabetes Mellitus (\%) & $7(11.9 \%)$ & $16(76.2 \%)$ & $2(9.0 \%)$ \\
\hline Hypertension (\%) & 16(27.1\%) & * $3(14.3 \%)$ & $10(45.5 \%)$ \\
\hline \multicolumn{4}{|c|}{ De Orio and Cofield's classification } \\
\hline Large (\%) & $56(94.9 \%)$ & $19(90.5 \%)$ & $15(58.2 \%)$ \\
\hline Massive (\%) & $3(5.1 \%)$ & $2(9.5 \%)$ & *7 (31.8\%) \\
\hline \multicolumn{4}{|l|}{ Surgical procedure } \\
\hline Suture bridge (\%) & $40(67.8 \%)$ & $14(66.7 \%)$ & $17(77.3 \%)$ \\
\hline Simgle row (\%) & $14(23.7 \%)$ & $4(19.0 \%)$ & $2(9.0 \%)$ \\
\hline Double row (\%) & $5(8.5 \%)$ & $3(14.3 \%)$ & $3(13.7 \%)$ \\
\hline LHB tenotomy (\%) & $29(49.2 \%)$ & $6(28.6 \%)^{*}$ & $13(59.0 \%)$ \\
\hline
\end{tabular}

Data are presented as mean \pm standard deviation unless otherwise indicated LHB, long head biceps

*Statistically significant $(P<.05)$ among the three groups

started 6 weeks after surgery, and isotonic muscle strengthening exercises began 12 weeks after surgery.

\section{Evaluation of functional outcome}

Functional outcome was evaluated preoperatively and postoperatively. The visual analog scale was used to measure pain (rest, night, and motion), the range of active motion was measured with a goniometer, muscle strength was measured with a handheld dynamometer (Micro FET2, Hoggan Health Industry, West Jordan, UT, USA), Japanese Orthopedic Association (JOA) score, and the University of California, Los Angeles (UCLA) score. An independent physiotherapist who was blinded to this study performed physical tests.

\section{Evaluation of structural outcome}

Acromiohumeral distance was evaluated, using the Oizumi classification [13], on plain radiographs that were taken with the patients standing and their arm held in a neutral position.

Tear length and width were measured on MRI using the protocol of Davidson et al. [14] FD of the supraspinatus, the infraspinatus/teres minor, and the subscapularis were evaluated on the most lateral oblique sagittal T2-weighted MRI with the scapular body (the "Y-view") $[15,16]$, using both Goutallier classification system and ImageJ [14]. The infraspinatus and teres minor were combined into a single measurement, because their borderline was not always clearly confirmed [17]. Muscle atrophy (MA) was evaluated using the relative ratio of the cross-sectional area of the subscapularis, supraspinatus, and infraspinatus/teres minor muscle belly to that of the supraspinatus fossa. For this measurement, we used ImageJ using the protocol of Nakamura et al. [18]

Retear of the rotator cuff was evaluated using Sugaya's classification [19]: type I, sufficient thickness and evenly low intensity; type II, sufficient thickness and heterogeneous high intensity; type III, repaired cuff tear that kept its continuity but had insufficient thickness; type IV, minor discontinuity and the torn area was minimal in the sagittal plane; and type $\mathrm{V}$, major discontinuity and torn area spread in the sagittal plane. Patients with types IV and V were admitted with postoperative retear [20]. An experienced, orthopedics-trained radiologist who was blinded to the study reviewed these images.

\section{Statistical analysis}

The statistical analysis was performed with JMP11 software (SAS, Cary, NC, USA). The Kruskal-Wallis test or $X^{2}$ test was used to compare the continuous or nominal variables in demographics and functional and structural outcomes among the three groups. A Wilcoxon test was used for comparing the preoperative and postoperative functional outcomes in each group. Spearman's $\rho$ was calculated to observe the nonparametric correlation of structural outcomes and clinical outcomes. The correlation between the data evaluated with Goutallier's classification and ImageJ was examined with Spearman's correlation coefficient. For identifying the risk factors for retear after surgery, univariate analysis was first performed in each 
group; then, multivariate logistic regression was performed using a step wise manner. A receiver-operating curve was calculated to detect the cut-off value when significance was noted in the multivariate analysis. The level of significance was defined for all calculations as $P<.05$. Data are expressed as a mean value with standard deviation.

\section{Results}

\section{Preoperative and postoperative functional outcome}

Preoperative JOA scores significantly improved from $57.9 \pm 19.9$ points preoperatively to $87.4 \pm 10.0$ points postoperatively in group AS, from $56.9 \pm 15.4$ points to $89.9 \pm 6.6$ points in group PS, and from $61.7 \pm 7.5$ points to $83.0 \pm 11.4$ points in group APE. Consistently, UCLA scores significantly improved from $18.2 \pm 5.0$ points preoperatively to $28.3 \pm 7.2$ points postoperatively in Group AS, from $17 \pm 4.7$ points to $29.4 \pm 3.8$ points in group PS, and from $15.9 \pm 4.0$ points to $28.3 \pm 5.9$ points in group APE. There were no significant differences of postoperative JOA/UCLA scores among the groups. The clinical outcomes scores are shown in Table 2.

Rest, motion, and night pain levels in the three groups were significantly improved postoperatively, except for rest pain in group APE, but it tended to have significance $(P=0.06)$. Most of the parameters in ROM and muscle strength in the three groups significantly improved or tended to have statistical significance after surgery.

\section{Preoperative structural outcome}

In the Oizumi classification, Class 0 was observed in 19, 6 , and 3 patients; Class I in 30,7 , and 8 patients; Class II in 8,7 , and 4 patients, and Class III in 2,1 , and 5 patients in Group AS, PS, and APE, respectively. Only two patients in group APE had Class IV.

The average retraction of the torn tendon was $29.1 \pm 6.2 \mathrm{~mm}$ in group AS, $31.2 \pm 10.4 \mathrm{~mm}$ in group PS, and $37.1 \pm 8.7 \mathrm{~mm}$ in group APE. The extent of the retraction was significantly larger in group PS and APE than group AS $(P=0.02)$. The average width of the torn tendon was $34.7 \pm 66.0 \mathrm{~mm}$ in group $\mathrm{AS}, 37.2 \pm 10.4 \mathrm{~mm}$ in group PS, and $45.7 \pm 89.8 \mathrm{~mm}$ in group APE. The extent of the width was significantly larger in group PS and APE than in group AS $(P=0.002)$.

The average MA in group AS was $246.5 \pm 83.5 \%$ in the subscapularis, $76.2 \pm 19.8 \%$ in the supraspinatus, and $220 \pm 51.9 \%$ in the infraspinatus/teres minor. For group PS, MA was $220.3 \pm 67.4 \%$ in the subscapularis, $78.9 \pm 18.6 \%$ in the supraspinatus, and $183.4 \pm 38.5 \%$ in the infraspinatus/teres minor; for group APE, MA was $252.1 \pm 82.4 \%$ in the subscapularis, $71.3 \pm 16 \%$ in the supraspinatus, and $187.4 \pm 54.8 \%$ in the infraspinatus/ teres minor.

The average FD, measured with Image J, for group AS was $5.35 \pm 8.25 \%$ in the subscapularis, $7.84 \pm 10.24 \%$ in the supraspinatus, and $3.35 \pm 4.92 \%$ in the infraspinatus/ teres minor; for group PS, FD was $3.5 \pm 5.2 \%$ in the subscapularis, $11.42 \pm 10.15 \%$ in the supraspinatus, and $8.52 \pm 9.23 \%$ in the infraspinatus/teres minor; and for group APE, FD was $6.0 \pm 7.23 \%$ in the subscapularis, $12.2 \pm 9.7 \%$ in the supraspinatus, and $6.05 \pm 5.2 \%$ in the infraspinatus/teres minor.

A low-grade Goutallier stage (stages 0 to 2) was seen in over $80 \%$ patients in all three groups. A high-grade Goutallier stage (stages 3 and 4) in group AS was seen in the subscapularis of two patients, supraspinatus of nine patients, and infraspinatus/teres minor of two patients; in Group PS, in the subscapularis of no patients, supraspinatus of three patients, and infraspinatus/teres minor of three patients. In Group APE, a high-grade Goutallier stage was seen in the subscapularis of two patients, supraspinatus of five patients, and infraspinatus/ teres minor of three patients. The global fatty degeneration index (GFDI) was $1.02 \pm 0.62$ in Group AS, $1.36 \pm 0.5$ in group PS, and $1.29 \pm 0.5$ in group APE. There was no significant difference among the three groups in GFDI (Table 3).

\section{Postoperative structural outcome}

Postoperative retear (Sugaya types IV and V) was noted in 26 of 102 patients (25.5\%) in this series: 10 patients in group AS (16.9\%), 9 patients in group PS (42.9\%), and 7 patients in group APE (31.8\%). The retear rate was

Table 2 Preoperative and postoperative clinical outcome in three groups

\begin{tabular}{|c|c|c|c|}
\hline & Group AS $(N=59)$ & Group PS $(N=21)$ & Group APE $(N=22)$ \\
\hline \multicolumn{4}{|l|}{ JOA score } \\
\hline Preoperative & $57.9 \pm 19.9(0-89.5)$ & $56.9 \pm 15.4(12-82)$ & $61.7 \pm 7.5(46-78.5)$ \\
\hline Postoperative & $87.4 \pm 10.0(65-100)$ & $89.9 \pm 6.6(80.5-99.5)$ & $83.0 \pm 11.4(60-96)$ \\
\hline$P$ value & $<0.001$ & $<0.001$ & $<0.001$ \\
\hline \multicolumn{4}{|l|}{ UCLA score } \\
\hline Preoperative & $18.2 \pm 5.0(7-31)$ & $17 \pm 4.7(9-26)$ & $15.9 \pm 4.0(8-23)$ \\
\hline Postoperative & $28.3 \pm 7.2(20-35)$ & $29.4 \pm 3.8(22-35)$ & $28.3 \pm 5.9(17-35)$ \\
\hline$P$ value & $<0.001$ & $<0.001$ & $<0.001$ \\
\hline
\end{tabular}

JOA Japanese Orthopedic Association, UCLA University of California, Los Angeles 
Table 3 Preoperative structural outcome in three groups

\begin{tabular}{|c|c|c|c|}
\hline & Group AS $(N=59)$ & Group PS $(N=21)$ & Group API: $(N=22)$ \\
\hline \multicolumn{4}{|c|}{ Oizumi classification } \\
\hline Grade O & $19(32.2 \%)$ & $6(28.6 \%)$ & $3(13.6 \%)$ \\
\hline Grade I & $30(50.8 \%)$ & 7 (33.3\%) & $8(36.4 \%)$ \\
\hline Grade II & $8(13.6 \%)$ & 7 (33.3\%) & $4(18.2 \%)$ \\
\hline Grade III & $2(3.4 \%)$ & $1(4.8 \%)$ & $5(22.7 \%)$ \\
\hline Grade IV & $0(0 \%)$ & $0(0 \%)$ & $2(9.1 \%)$ \\
\hline Retraction (mm) & $29.1 \pm 6.2$ & $31.2 \pm 10.4$ & $37.1 \pm 8.7$ \\
\hline Width (mm) & $34.7 \pm 6.0$ & $37.2 \pm 10.4$ & $45.0+9.8$ \\
\hline \multicolumn{4}{|c|}{ Muscle atrophy (\%) } \\
\hline SSC & $246.5 \pm 83.5$ & $220.3 \pm 67.4$ & $252.1 \pm 82.4$ \\
\hline SSP & $76.2 \pm 19.8$ & $78.9 \pm 18.6$ & $71.3 \pm 16$ \\
\hline ISP/TM & $220.4 \pm 51.9$ & $183.4 \pm 38.5$ & $187.4 \pm 54.8$ \\
\hline \multicolumn{4}{|c|}{ Fatty degeneration (\%) } \\
\hline SSC & $5.35 \pm 8.25$ & $3.5 \pm 520$ & $6.0 \pm 7.23$ \\
\hline SSP & $7.84 \pm 10.24$ & $11.42 \pm 10.15$ & $12.2 \pm 9.7$ \\
\hline ISP/IM & $3.35 \pm 4.92$ & $8.52 \pm 9.23$ & $6.05 \pm 5.2$ \\
\hline \multicolumn{4}{|c|}{ Goutallier classification SSC } \\
\hline Stage 0 & $24(40.7 \%)$ & $9(42.9 \%)$ & $6(27.3 \%)$ \\
\hline Stage 1 & $26(44.1 \%)$ & $8(38.1 \%)$ & $5(22.7 \%)$ \\
\hline Stage 2 & 7 (11.9\%) & $4(19.0 \%)$ & $9(40.9 \%)$ \\
\hline Stage 3 & $2(3.4 \%)$ & $0(0 \%)$ & $1(4.5 \%)$ \\
\hline Stage 4 & $0(0 \%)$ & $0(0 \%)$ & $1(4.5 \%)$ \\
\hline \multicolumn{4}{|c|}{ Goutallier classification SSP } \\
\hline Stage 0 & $13(22 \%)$ & $2(9.5 \%)$ & $2(9.0 \%)$ \\
\hline Stage 1 & $16(27.1 \%)$ & $5(23.8 \%)$ & $5(22.7 \%)$ \\
\hline Stage 2 & $21(35.6 \%)$ & $11(52.4 \%)$ & $10(45.5 \%)$ \\
\hline Stage 3 & 7 (11.9\%) & $2(9.5 \%)$ & $1(4.5 \%)$ \\
\hline Stage 4 & $2(3.4 \%)$ & $1(4.8 \%)$ & $4(18.2 \%)$ \\
\hline \multicolumn{4}{|c|}{ Goutallier classification ISP/TM } \\
\hline Stage 0 & $28(47.5 \%)$ & $5(22.7 \%)$ & $5(22.7 \%)$ \\
\hline Stage 1 & $26(44.1 \%)$ & $6(27.3)$ & $9(40.9 \%)$ \\
\hline Stage 2 & $3(5.0 \%)$ & $7(31.8 \%)$ & $5(22.7 \%)$ \\
\hline Stage 3 & $2(3.4 \%)$ & $3(13.6 \%)$ & $1(4.5 \%)$ \\
\hline Stage 4 & $0(0 \%)$ & $0(0 \%)$ & $2(9.0 \%)$ \\
\hline GFDI & $1.02 \pm 0.62$ & $1.36+0.5$ & $1.29 \pm 0.5$ \\
\hline
\end{tabular}

SSC subscapularis, SSP supraspinatus, ISP/TM infraspinatus/teres minor, GFDI global fatty degeneration index

significantly higher in group PS than in groups AS and APE $(P=0.02)($ Table 4$)$.

\section{Surgical technique and postoperative retear}

Suture bridge technique was performed in 71 patients: 40 in group AS;14 in group PS; 17 in group APE. Postoperative retear occurred in 16 patients (22.5\%): 6 in group AS (15.0\%); 4 in group PS (28.6\%); 6 in group APE (35.3\%).
Single-row technique was performed in 20 patients: 14 in group AS; 4 in group PS; 2 in group APE. Postoperative retear occurred in 8 patients (40.0\%): 4 in group AS (28.6\%); 3 in group PS (75.0\%); 1 in group APE (50.0\%).

Double-row technique was performed in 11 patients: 5 in group AS; 3 in group PS; 3 in group APE. Postoperative retear occurred in 2 patients (18.2\%): none in group AS (0.0\%); 2 in group PS (66.7\%); none in group APE (0.0\%). Details are shown in Table 5. 
Table 4 Postoperative retear (SUGAYA's classification)

\begin{tabular}{lllll}
\hline SUGAYA & Group AS $(N=59)$ & Group PS $(N=21)$ & Group APE $(N=22)$ & Total \\
\hline Type I & $28(45.8 \%)$ & $8(38 \%)$ & $3(13.6 \%)$ \\
Type II & $13(22 \%)$ & $2(9.5 \%)$ & $6(27.3 \%)$ & $3(13.6 \%)$ \\
Type III & $8(13.6 \%)$ & $2(9.5 \%)$ & $4(18.2 \%)$ & 15 \\
Type IV & $8(13.6 \%)$ & $4(19 \%)$ & $7(31.8 \%)$ & $26(25.5 \%)$ \\
Type V & $2(3.4 \%)$ & $5(23.8 \%)$ & $9(42.9 \%)$ &
\end{tabular}

\section{Risk factors affecting postoperative retear}

First, various parameters were evaluated to determine the risk factors for retear after surgery, using univariate analysis. Retraction $(P=0.039)$, width $(P=0.0023)$, FD of the supraspinatus $(P=0.0043)$, the Goutallier classification of the supraspinatus $(P=0.001)$, and GFDI $(P=0.008)$ were significant risk factors in group AS: Preoperative active external rotation range $(P=0.001)$, preoperative muscle strength of flexion $(P=0.02)$, and FD of the infraspinatus/teres minor $(P=0.048)$ were evaluated using ImageJ in group PS. FD of the supraspinatus $(P=0.002)$ and the infraspinatus/teres minor $(P=0.0074)$ were evaluated using ImageJ and GFDI $(P=0.0123)$ in group APE. The Goutallier stage of the infraspinatus in group PS and APE was not a significant risk factor, but it tended to have statistical significance (Table 6).

Next, multivariate analysis using stepwise methods was performed. Preoperative external rotation range was the only risk factor for postoperative retear in groups PS and APE $(P=0.014$ and $P=0.016$, respectively). For the prediction of postoperative retear, receiver operating characteristic (ROC) curve analysis demonstrated that the cut-off value in the preoperative external rotation range was $25^{\circ}$, showing that the retear risk increased 2.12 -fold as the preoperative external rotation range decreased by $5^{\circ}$ (Fig. 1 ).

Table 5 Univariate analysis in three groups

\begin{tabular}{clll}
\hline & Healed $(N=76)$ & Retear $(N=26)$ & Total $(N=102)$ \\
\hline Suture bridge & $55(77.5 \%)$ & $16(22.5 \%)$ & 71 \\
Group AS & $34(85.0 \%)$ & $6(15.0 \%)$ & 40 \\
Group PS & $10(71.4 \%)$ & $4(28.6 \%)$ & 14 \\
Group APE & $11(64.7 \%)$ & $6(35.3 \%)$ & 17 \\
Single row & $12(60.0 \%)$ & $8(40.0 \%)$ & 20 \\
Group AS & $10(71.4 \%)$ & $4(28.6 \%)$ & 14 \\
Group PS & $1(25.0 \%)$ & $3(75.0 \%)$ & 4 \\
Group APE & $1(50.0 \%)$ & $1(50.0 \%)$ & 2 \\
Double row & $9(81.8 \%)$ & $2(18.2 \%)$ & 11 \\
Group AS & $5(100 \%)$ & $0(0 \%)$ & 5 \\
Group PS & $1(33.3 \%)$ & $2(66.7 \%)$ & 3 \\
Group APE & $3(100 \%)$ & $0(0 \%)$ & 3 \\
\hline
\end{tabular}

\section{Correlation between active external rotation range and its related variables}

Since multivariate analysis showed that active external rotation range (AERR) is a unique risk factor for postoperative retear in groups PS and APE, we further evaluated the correlation between AERR and its related variables in these groups. There was statistical significance between AERR and FD, using Image $(r=-0.36$, $P=0.04)$. External rotation strength (ERS) was not significant, but it showed a trend $(P=0.06)$. For FD of the infraspinatus, statistical significance was seen between the evaluation methods, using the Goutallier classification and ImageJ $(r=0.82, P<0.0001)$ (Table 7$)$.

\section{Discussion}

The present study investigated the risk factors for retear after ARCR in large and massive cuff tears, dividing these tears into three groups (i.e., group AS, PS, and APE). Although univariate analysis revealed that the groups had different characteristics, step-wise multivariate analysis showed that preoperative, decreased active external rotation in group PS and APE was a unique risk factor for retear after surgery, with a cut-off value of $25^{\circ}$. To our knowledge, such data have not been reported.

Previous studies that used multivariate analysis demonstrated that the Goutallier stage of the infraspinatus is a risk factor for postoperative retear in large and massive tears $[8,21]$. In the present study, the Goutallier stage of the infraspinatus in Group PS and APE was a significant factor for postoperative retear in univariate analysis, but not in multivariate analysis. The average Goutallier stage of the infraspinatus was relatively low (0.9) in the present study, compared with 1.2 in a study by Oh et al. [8] and 2.1 in a study by Chung et al. [21] Thus, this may partly explain why the Goutallier stage of the infraspinatus did not reach statistical significance in the present study.

FD of the infraspinatus caused postoperative retear and led to limitations of external rotation [22]. Loss of active external rotation is related to tears in the infraspinatus and teres minor [23]. In the present study, there was a significant correlation between the decrease of active external rotation range and FD, evaluated with ImageJ. Taken together, these results supported our data that decreased 
Table 6 Correlation between active external rotation range (AERR) and its related variables

\begin{tabular}{|c|c|c|c|c|c|}
\hline Group AS $(N=59)$ & $P$ value & Group PS $(N=21)$ & $P$ value & Group APE $(N=22)$ & $P$ value \\
\hline Retraction & 0.039 & Preoperative ER & 0.001 & SSP FD & 0.002 \\
\hline Width & 0.0023 & Preoperative FLEX MS & 0.02 & ISP/TM FD & 0.0074 \\
\hline SSP FD & 0.0043 & ISP/TM FD & 0.048 & GFDI & 0.012 \\
\hline Goutallier SSP & 0.001 & & & & \\
\hline GFDI & 0.0084 & ${ }^{*}$ Goutallier ISP & 0.08 & ${ }^{*}$ Goutallier ISP & 0.068 \\
\hline
\end{tabular}

$E R$ external rotation, MS muscle strength, FD fatty degeneration, GFDI global fatty degeneration index

*There were no significant differences or trends

active external range is significantly associated with postoperative retear in patients who undergo ARCR for treatment of large or massive tears.

In the present study, multivariate analysis showed that decreased active external range before surgery was a risk factor for retear in group PS and APE. The preoperative characteristics in these two groups revealed a similar tendency, except for distribution of large or massive tears. Thus, less fatty involvement of the subscapularis in the two groups may have contributed to the similar data.

In AS cuff tears, the retear rate after surgery was reported to be $6-18 \%$ [24-26]. Consistent with these studies, our study found 10 postoperative retear cases $(16.9 \%, N=59$ cases) in group AS. A previous study found that the Goutallier stage of the subscapularis was associated with postoperative retear in AS cuff tears [25], while the Goutallier stage of the supraspinatus was responsible for retear after ARCR in the present study. A high-grade Goutallier stage was found in the supraspinatus in nine cases $(15.3 \%)$ and in the subscapularis in two cases $(3.4 \%)$ in group AS. Thus, this might have

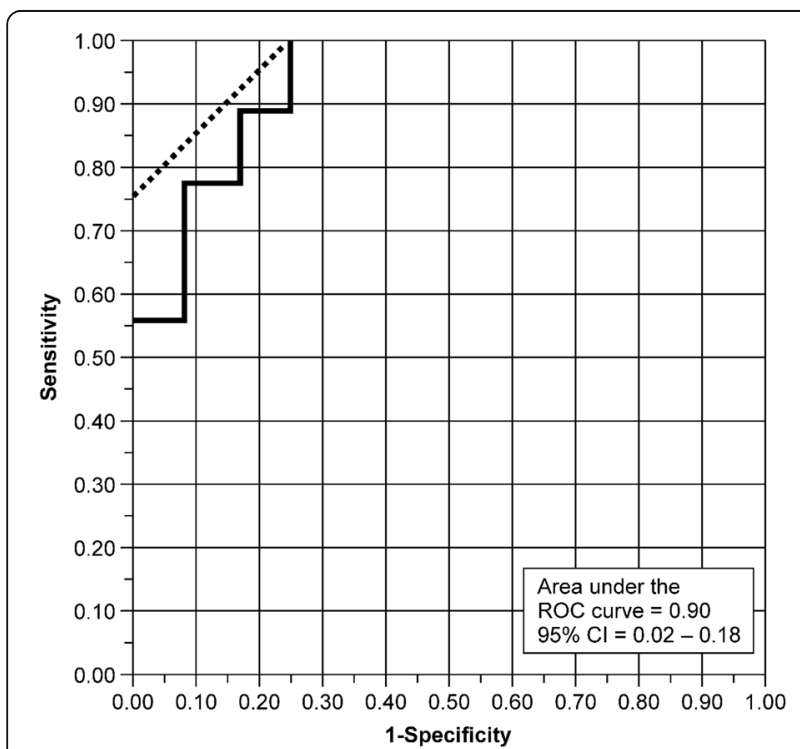

Fig. 1 Receiver operating characteristic (ROC) curve analysis to calculate the cutoff value for preoperative active external rotation range. AUC area under the curve, Cl confidence interval affected our data. Although univariate analysis in the present study showed a certain risk for postoperative retear in anteroposterior cuff tears, no significant factors were noted in the multivariate analysis. Studies on these points are now underway at our institution.

Although most tears occurred in the supraspinatus tendon, tearing in this tendon did not influence retear after surgery in group PS and APE. Mochizuki et al. [27, 28] reported that the footprint of the supraspinatus tendon on the greater tuberosity is much smaller than previously believed, and this area of the greater tuberosity is actually occupied by a substantial amount of the infraspinatus tendon. This may mean that during surgery, infraspinatus tendon repair rather than supraspinatus tendon repair may be closely associated not only with the footprint coverage at the greater tuberosity, but also with retear after surgery at the site.

The limitations of the present study were its retrospective cohort, short-term follow up, and small sample size, especially in group PS and APE in comparison with group AS. Further studies with longer follow-up and larger cohorts are needed to address these limitations. However, the strength of this study was that we clearly demonstrated that decreased active eternal range is a risk factor for large and massive tears, especially in PS and APE cuff tears.

\section{Conclusions}

Although multivariate analysis failed to detect significant risk factor for retear in patients with anterosuperior large/ massive cuff tears who undergo ARCR, it demonstrated

Table 7 Correlation between active external rotation range (AERR) and its related variables

\begin{tabular}{llll}
\hline & & Correlation coefficient $(r)$ & $P$ Value \\
\hline Preoperative ER & Preoperative ER MS & 0.3 & 0.06 \\
Preoperative ER & ISP FD & -0.36 & 0.04 \\
Preoperative ER & Goutallier ISP & -0.154 & 0.37 \\
ISP FD & Preoperative ER MS & -0.0154 & 0.93 \\
ISP FD & Goutallier ISP & 0.82 & $<0.001$ \\
Goutallier ISP & Preoperative ER MS & 0.05 & 0.77 \\
\hline
\end{tabular}

ER external rotation, $M S$ muscle strength, ISP infraspinatus 
that active external rotation less than $25^{\circ}$ before surgery is a significant risk factor in those with posterosuperior large/massive tears. This study may help surgeons understand the results of arthroscopic surgery in patients with large/massive tears.

\section{Abbreviations}

AERR: Active external rotation range; ARCR: Arthroscopic rotator cuff repair; AUC: Area under the curve; ERS: External rotation strength; FD: fatty degeneration; GFDI: The Global Fatty Degeneration Index; JOA: Japanese Orthopedic Association; MA: Muscle atrophy; MRI: Magnetic resonance imaging; RCTs: Rotator cuff tears; ROC: Receiver-operating characteristic; ROM: Range of motion; UCLA: The University of California, Los Angeles

\section{Acknowledgements}

We thank Tatsuyuki Kakuma PhD from the Department of Bio-Statistical Center, Kurume University for his help with the statistical analysis.

\section{Funding}

We have no funding.

\section{Availability of data and materials}

All data supporting the results reported in a published article can be found in the reference section.

\section{Authors' contributions}

HS planned the study's design, data acquisition, analysis, and interpretation of data, and wrote the manuscript. MG supervised the study design, data analysis, and manuscript writing. YM, TO, and NS supervised the study design and data analysis. $\mathrm{HH}$ and $\mathrm{HN}$ carried out data acquisition and analysis. TK carried out the statistical data analysis. All authors read and approved the final manuscript.

\section{Ethics approval and consent to participate}

This retrospective study was approved by the authorized institutional review board at the Ethical Committee of Kurume University (approval no. 12333).

\section{Consent for publication}

All consents to publish from the patients took part in this study were obtained.

\section{Competing interests}

The authors declare that they have no competing interests.

\section{Publisher's Note}

Springer Nature remains neutral with regard to jurisdictional claims in published maps and institutional affiliations.

\section{Author details}

'Department of Orthopaedic Surgery, Kurume University School of Medicine, 67 Asahi-machi, kurume, Fukuoka 830-0011, Japan. Department of Statistics, Kurume University School of Medicine, 67 Asahi-machi, kurume, Fukuoka 830-0011, Japan. ${ }^{3}$ Department of Orthopaedic Surgery, Kurume University Medical Center, 155-1 Kokubu-machi Kurume, Fukuoka 839-0863, Japan.

Received: 10 June 2017 Accepted: 18 September 2017

Published online: 25 September 2017

References

1. Berth A, Neumann W, Awiszus F, Pap G. Massive rotator cuff tears: functional outcome after debridement or arthroscopic partial repair. J Orthop Traumatol. 2010;11:13-20.

2. Burkhart SS, Barth JR, Richards DP, Zlatkin MB, Larsen M. Arthroscopic repair of massive rotator cuff tears with stage 3 and 4 fatty degeneration. Arthroscopy. 2007:23:347-54.

3. Burkhart SS, Danaceau SM, Pearce CE Jr. Arthroscopic rotator cuff repair: Analysis of results by tear size and by repair technique-margin convergence versus direct tendon-to-bone repair. Arthroscopy. 2001;17:905-12.
4. Galatz LM, Ball CM, Teefey SA, Middleton WD, Yamaguchi K. The outcome and repair integrity of completely arthroscopically repaired large and massive rotator cuff tears. J Bone Joint Surg Am. 2004;86-A:219-24.

5. Lo IK, Burkhart SS. Arthroscopic revision of failed rotator cuff repairs: technique and results. Arthroscopy. 2004;20:250-67.

6. Yoo JC, Ahn JH, Koh KH, Lim KS. Rotator cuff integrity after arthroscopic repair for large tears with less-than-optimal footprint coverage. Arthroscopy. 2009;25:1093-100

7. Saccomanno MF, Sircana G, Cazzato G, Donati F, Randelli P, Milano G. Prognostic factors influencing the outcome of rotator cuff repair: a systematic review. Knee Surg Sports Traumatol Arthrosc. 2015; https://doi. org/10.1007/s00167-015-3700-y.

8. Oh JH, Kim SH, Ji HM, Jo KH, Bin SW, Gong HS. Prognostic factors affecting anatomic outcome of rotator cuff repair and correlation with functional outcome. Arthroscopy. 2009:25:30-9.

9. Kim JR, Cho YS, Ryu KJ, Kim JH. Clinical and radiographic outcomes after arthroscopic repair of massive rotator cuff tears using a suture bridge technique: assessment of repair integrity on magnetic resonance imaging Am J Sports Med. 2012:40:786-93.

10. Ueda Y, Sugaya H, Takahashi N, Matsuki K, Kawai N, Tokai M, et al. Rotator cuff lesions in patients with stiff shoulders: a prospective analysis of 379 shoulders. J Bone Joint Surg Am. 2015;97:1233-7.

11. Warner JJ, Higgins L, Parsons IM, Dowdy P. Diagnosis and treatment of anterosuperior rotator cuff tears. J Shoulder Elbow Surg. 2001;10:37-46.

12. DeOrio JK, Cofield RH. Results of a second attempt at surgical repair of a failed initial rotator-cuff repair. J Bone Joint Surg Am. 1984;66:563-7.

13. Miyoshi N, Suenaga N, Katayama K, Oizumi N, Yamaguchi H, Matsuno T. Radiological classification of glenoid deformity in rheumatoid arthritis. Int J Rheumatol. 2011;2011:239894.

14. Davidson JF, Burkhart SS, Richards DP, Campbell SE. Use of preoperative magnetic resonance imaging to predict rotator cuff tear pattern and method of repair. Arthroscopy. 2005:21:1428.

15. Mellado JM, Calmet J, Olona M, Esteve C, Camins A, Perez Del Palomar L, et al. Surgically repaired massive rotator cuff tears: MRI of tendon integrity, muscle fatty degeneration, and muscle atrophy correlated with intraoperative and clinical findings. AJR Am J Roentgenol. 2005;184:1456-63.

16. Lapner PL, Jiang L, Zhang T, Athwal GS. Rotator cuff fatty infiltration and atrophy are associated with functional outcomes in anatomic shoulder arthroplasty. Clin Orthop Relat Res. 2015;473:674-82.

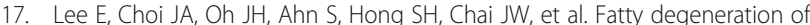
the rotator cuff muscles on pre- and postoperative CT arthrography (CTA): is the Goutallier grading system reliable? Skelet Radiol. 2013;42:1259-67.

18. Nakamura H, Gotoh M, Mitsui $Y$, Honda H, Ohzono H, Shimokobe $H$, et al. Factors affecting clinical outcome in patients with structural failure after arthroscopic rotator cuff repair. Arthroscopy. 2016;32:732-9.

19. Sugaya H, Maeda K, Matsuki K, Moriishi J. Repair integrity and functional outcome after arthroscopic double-row rotator cuff repair. A prospective outcome study. J Bone Joint Surg Am. 2007;89:953-60.

20. Choi S, Kim MK, Kim GM, Roh YH, Hwang IK, Kang H. Factors associated with clinical and structural outcomes after arthroscopic rotator cuff repair with a suture bridge technique in medium, large, and massive tears. J Shoulder Elbow Surg. 2014;23:1675-81.

21. Chung SW, Kim JY, Kim MH, Kim SH, Oh JH. Arthroscopic repair of massive rotator cuff tears: outcome and analysis of factors associated with healing failure or poor postoperative function. Am J Sports Med. 2013;41:1674-83.

22. Goutallier D, Postel JM, Bernageau J, Lavau L, Voisin MC. Fatty muscle degeneration in cuff ruptures. Pre- and postoperative evaluation by CT scan Clin Orthop Relat Res. 1994;304:78-83.

23. Collin P, Matsumura N, Ladermann A, Denard PJ, Walch G. Relationship between massive chronic rotator cuff tear pattern and loss of active shoulder range of motion. J Shoulder Elbow Surg. 2014;23:1195-202.

24. Schnaser E, Toussaint B, Gillespie R, Lefebvre Y, Gobezie R. Arthroscopic treatment of anterosuperior rotator cuff tears. Orthopedics. 2013;36:e1394-400.

25. Maqdes A, Abarca J, Moraiti C, Boughebri O, Dib C, Leclere FM, et al. Does preoperative subscapularis fatty muscle infiltration really matter in anterosuperior rotator cuff tears repair outcomes? A prospective multicentric study. Orthop Traumatol Surg Res. 2014:100:485-8.

26. Ide J, Tokiyoshi A, Hirose J, Mizuta H. Arthroscopic repair of traumatic combined rotator cuff tears involving the subscapularis tendon. J Bone Joint Surg Am. 2007;89:2378-88. 
27. Mochizuki T, Sugaya H, Uomizu M, Maeda K, Matsuki K, Sekiya I, et al. Humeral insertion of the supraspinatus and infraspinatus. New anatomical findings regarding the footprint of the rotator cuff. J Bone Joint Surg Am. 2008:90:962-9.

28. Mochizuki T, Sugaya H, Uomizu M, Maeda K, Matsuki K, Sekiya I, et al. Humeral insertion of the supraspinatus and infraspinatus. New anatomical findings regarding the footprint of the rotator cuff. Surgical technique. J Bone Joint Surg Am. 2009;91(Suppl 2 Pt 1):1-7.

Submit your next manuscript to BioMed Central and we will help you at every step:

- We accept pre-submission inquiries

- Our selector tool helps you to find the most relevant journal

- We provide round the clock customer support

- Convenient online submission

- Thorough peer review

- Inclusion in PubMed and all major indexing services

- Maximum visibility for your research

Submit your manuscript at www.biomedcentral.com/submit 